\title{
LATE STAGES OF MASSIVE STAR EVOLUTION AND NUCLEOSYNTHESIS
}

Ken ichi Nomoto and Masa-ałi Hashimoto

\author{
Department of Physics, Brookhaven National Labnratory \\ Upton, NY 11973, U.S.A.
}

on leave from the Department of Earth Science and Astronomy College of Arts and Sciences, University of Tokyo

Meguro-ku, Tokyo 153, Japan

A lecture delivered at International School of Nuclear Physics, 10th Course The Early Universe and Its Evolution Erice, April 2-14, 1986

to be published in Progress in Particle and Nuclear Physics, Vol 18, 1986

\section{DISCLAIMER}

This repor: was prepared as an account of wotk sponsored by an agency of the United States Government. Neither the United States Government jor any agency therew, jor thy of their employees, makes any warranty, express or implied, or assumes any legal liability or responsibility for the accuracy, completeness, or usefulness of any jnformation, upparatus, product, or process disclosed, or Iepresents that its use would not infringe privately owned rights. Reference hetein to any specific commercial product, process, or scriice by trade name, irademark, manufacturer., of otherwise does not recessarily constitute or imply its endorsement, tecommendation, or fayoring by the United Stales Government or any agency thereor, The wiews and opiniors of authors expresed therein do not necessarily state or reflect those of the United States Government or any agency thereor. 


\title{
LATE STAGES OF MASSIVE STAR EVOLUTION AND NUCLEOSYNTIJESIS
}

\author{
Ken'ichi Nomoto and Mastaki liasthituto \\ Department of Physics, Brookhaven National Laboratory \\ Upton, NY 11073, U.S.A. \\ on leave from the Departanest of Eatth Science and Astronomy \\ College of Atts and Sciences, University of Tokyo \\ Ileguro-ku, Tokyo 153, Japan
}

\begin{abstract}
The evolution of massive stars in the mass range of $8-25 \mathrm{M} / \mathrm{S}$ is reviewed. The effect of electron degencracy on the gravothermal satuse of stirs is diseussed. Depending on the stellar mess, the stars form three types of cores, namely, non-degenerate, semi-degencrate, and atrongty degenerate cores. The evolution for these cases is quite distinct from each other and leads to the three different types of final fate. It is suggested that our helium star model, which is equivalent to a $25 M_{\odot}$ star, will form a relatively small mass iton core despite the faster ${ }^{12} \mathrm{C}(\alpha, \gamma){ }^{16} \mathrm{O}$ reaction.
\end{abstract}

\section{KEYWORDS}

Stellar evolution; gravothermodynamics; nucleosynthesis; supernovae.

\section{NTIRODUCTION}

Big Bang rucleosynthesis produces helium and some cther light elements bit not beyond, because ${ }^{3} \mathrm{Be}$ is unstable and the expansion of the universe is too fast and its density is too low to synthesize ${ }^{2} \mathrm{C}$ through the $3 \alpha$ reaction (e.g., Schramm 1986 for a recent seview). In other words, the decrense in tempernture is much faster than nuclear zeacticns that tend to realize nuclear statistical equilibrium (NSE) for the corresponding temperature.

However, the universe provides another site of nucleosynthesis. As a zesult of decreasing temperature, gravitational forces become effective and form stars (before or after the galaxy formation). Stars then evolve. Their internal temperature increases, not decreases, and eventually reaches the ignition temperature of nuclear burning. Starting from hyirogen burning, helum, carbon, oxygen, reon, and silicos burn successively and synthesize heavier elements as the interio: temperature continues to increase. This process is considered to be approaching iNE and eventually iron peak elements are synthesized. Finally those elements are ejected from stars into space by means of stellar wind and i supernova explosion. This is the origin of heavy elements. 
In construeting the theory of element synthesis, the key is to understand why and how stars evolye, or why stars increase their interior temperature and density and develop a complex structure. Therefore we start from the thermodynamics of self-gravitatisg system, i.e, gravothermodynamies (Sugimoto et al, 1981), which is described in the next seetion. The actual evolution of non-degenerate nassive stars ( $M T 25$ $\left.M_{\odot}\right), 10-13 M_{\odot}$ stars which develop scui-degcrerate cores, and 8 - $10 . M_{\odot}$ stars whish forn strongly vection degenerate cores, are discussed in $5.4-56$, zespectively.

\section{GRAVOTHERMODYYAMICS AND ENOLUTION OF STARS}

Let us consider a systen that is governed by its own self-gravity ard is in hydrostatic equilibritu. Such a system will evcatually urdergo the so-called gravothermal catastrophe if the density contrawt $p_{c} / p_{1}$ between the center and the outer edge of the system is larger than a certain eritica) value (see Sugimoto et al. 1981 and references therein). For example, if $\rho_{e} / \rho_{1}>709$, an isothermal sphere surtounded by an adiabatic wall is secularly urstabe in the following way (Antonos 1360; Lynder-Bell and Wood 1908): If heat is transported from the center to the outer layers, the central region contracts to maintain the hydrostatic equilibrium. Then the central temperature, $T_{e}$, jucreases because gravitational energy telease dominates the loss of heat. The resulting temperature gradient promotes more heat flow from the center that induces further gravitational contraction and increase in $T_{e}$. The system is said to have negative specific heat because heat loss causes a temperature inctease. In other words, the systern carrot stay in thermal equilibritam but will move out of equilibrium with increasing $T_{c} / T_{1}$ ard $p_{c} / p_{1}$.

Such a mechanism to create a contrast of density and temperature is ensily understood from the behavior of stars which undergo quasi-static gravitational contraction. The jydrostatic stellar structure gives a geiation between $P_{\mathrm{c}}, p_{\mathrm{c}}$, and stellar mass, $M$, as

$$
\frac{P_{c}^{3}}{p_{c}^{4}}=4 \pi G^{3}\left(\frac{M I}{\phi}\right)^{2}
$$

Here $\phi$ is the non-dimensional mass and $\phi=10.73$ and 10.14 for the polytrope of index $\gamma=1.5$ and 3.0 , respectively (Sugiznoto and Nomoto 1960; also Arnett 1978). For an ideal gas, Eq.(1) gives

$$
\frac{T_{c}^{3}}{p_{c}} \sim M^{2}
$$

The specific entropy, $s$, is given as

$$
\left(\frac{\mu H}{k}\right) s_{e}=\ln \left(\frac{T_{e}^{3 / 2}}{\rho_{e}}\right)+C_{1}=\ln \left(\frac{M^{2}}{T_{c}^{3 / 2}}\right)+C_{2}=\ln \left(\frac{M}{\rho_{c}^{1 / 2}}\right)+C_{3},
$$

where $\mu$ is the mean molecular weight, $H$ atomic mass unit, $k$ Boltsmann constant, and $C_{1}, C_{2}$, ard $C_{3}$ additional constasts. Equations (2) and (3) show that for the same $T_{k}$ (i.e, soughly for the same guclear burning stage), $s_{c}$ is higher (and $\rho_{c}$ is lower) for larger $M$. We need higher entropy to sustrin a larger mass against self-gravity.

Another important feature of the stars is that they are open system losing energy by radiation and neutrinos. When nuclear reactions are not active, the star loses entropy, i.e., $s_{c}$ decreases. From $E_{q} .(3)$, we see that both $T_{c}$ and $\rho_{c}$ increase as $s_{c}$ decreases for fixed $M$. Ir other words, the specifie heat in the central region is negative

$$
c_{g} \equiv \frac{d s_{c}}{d \ln T_{c}}=-\frac{3}{2} \frac{k}{\mu H}
$$


The increase in $p_{c}$ implies tiat tje star contracts and zeleases gravitational energy, a part of which goes into internal energy and the rest goes inte radiation and neutrino losses. This is the renson why the interior temperature increases during stellar evolution.

As the temperature inercases, it renches the irgition temperature of nuclear burning. Then the star stays in

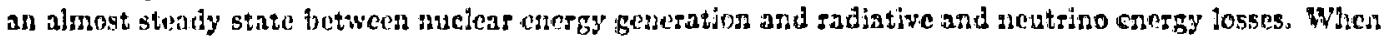
the nuclear fud is uxhinsted, the ashes forms a core and nuelens shell burning becomes active around the core. The core undegoes gravitational contraction to dnerease $p_{c}$ and $T_{c}$. On the other hame, the burning shell (denoted by the subeript 1) does not follow the corc contraction because, to proride the

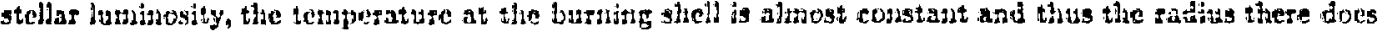
not appreciably cliange. As a sesult, $p_{\mathrm{e}} / \rho_{1}$ becomes so large that the cose edge can be fegurded as a stellar

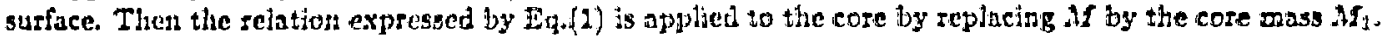
The core can be regarded as a single star (Hayashiet al. 1972). (The above role of the active burnitrg shell is crucial when stars evolve to red-giants. See Sugimoto and Nomoto 1980 for more detail.) The smaller $M M_{1}$ than $M_{\mathrm{f}}$ is consistent with smaller $s_{c}$ than that for a previous stage. Furtiner decrease in $s_{c}$ again leads to increase in $T_{c}$ until the asth is ignited. The cycle of graritational contraction and nuclear burnisg Jesids to the formation of an onion-like chemical structure as will be shown in later sections.

In short, the star evolves because it is a self-gravitating system and has a negative specific heat. The sign of the specfic heat depends on the equation of state, however. If the estopy is sufficiently thigh, the pressure depends on temperature. The decrease in entropy reiults in decrense in temperature and thus pressure, if density is fixed. Then, to maintain lydrostatic engilibrium, the star has to contract to increase the density and temperature. This situation is cumpletcly opposite if electrons are degenerate and the pressure dons not inuch depend on temperatuse, In this case, the Joss of entropy noes not auth affect the hydrostntie equilibrium and thus does not induce gravitational contraction. Accondingly $T_{E}$ decteases as qhe star loses energy. In other words, the star has a prositive spechfic heat and eventually becomes a cold white dwarf. $\mathbb{B} y$ applying the equation of state for non-relativistic degenerate electrons, $P=\boldsymbol{K}_{4}, \rho^{5 / 3}$, wo $\mathrm{Eq},(1)$, we obtaits a relation,

$$
p_{\varepsilon} \sim M^{2}
$$

If electrons are relativistically degenerate, i.e., $P=K_{2} \rho^{4 / 3}, \mathrm{Iq}_{\mathrm{q}}$ (1) gives the Chandrasekhar mass. Suct a change in equation of state occurs for stars with sufficiently low central entopy and thus for a suffieiently small mass, $M$, or small core mass, $M I_{1}$, as seen from $E q$. (3). Of cource, $M$ or $M_{1}$ should be smalles than the Chandrasekhar mass.

The example of the evolutionny cilange in the central density and temperature during the gravitational contraction is shown in Figures 1 and 2 for reon stars with masses $1.30-1.45 M_{0}$ (Nomoto $1984 a$, 1986). These evolutionary paths follow the reiation given in Eq.(1) for fixed $M$. At the peak temperature, the gravothermal specific heat changes its sign because electrons become strongly degenerate. The penk temperature is hitginer for larger. $M$ as seen in Figure 3 (Nomoto 1984a) because larger masses correspond to higher $T_{c}$ for the same $p_{e}$ in the non-degenerate regine (Eq. 2) and higher central densities for the degenerate regime ( $E \mathrm{~g} .5)$. If $M I$ is so small that the peak temperature is lower than the ignition temperature of the nuclear fuel, the stat will become a white dwarf. On the other hand, if $M$ is large enough, $T_{c}$ can reach the ignition temperature. Therefore, there exists a minimum mass for nuclear burning to oceur. The minimum mass is $0.08,0.25,1.06$, and $1.37 M_{\odot}$ for hydrogen, helium, carbon, and geon burning, respectively (e.g., Arnett 1978; Nomoto 1981 for a review).

Therefore, stars with a core mass, $M_{1}$, smaller than the abowe minimum mass will gict undergo further nuclear burning, but form a white dwarf-like degenerate core. In this sense, the stellar mass, M, is a critical parameter to determine the final fate of evolution and the stellar evolution is classified by Af as follows: 
1) For $M<0.08 M S_{\odot}$, the star will becone a planet-like black dwarf without agniting hydrogen butning.

2) For $0.08 M_{\odot}<M<0.45 M_{\odot}$, the star will crd up as a helium white dwarf, though such a single star is still a red-dwarf on the main-sequence because hydiogen cannot be depleted within a Hubble time.

3) For $0.45 M f_{\odot}<M<8 M M_{\odot}$, the star forms a degenerate $C+O$ core. Most of them will become a $C+O$ white dwarf by losing their hydrogen-rich envelope, Jut some of them $\left(\sim B-B M_{\odot}\right)$ could reach a supernova stage by increasing the $C+O$ eore mass to the Chandrasckins noss and ignites a carbon deflagration. The mechanisu is the sane as of Type Ia supornovae but, because of the existese of a fydrogerz-tich enwelope, the explosion will look like a Type II-L supernova (Doggett and Branch 1985).

4) For $B-10 M$ range, a degenerate $O+N e+M g$ core is formed. The star will evertually collapse due to clectron capture on ${ }^{24} \mathrm{Mg}$ and $20 \mathrm{Ne}$.

5) For 10 - $13 M$ ange, neon is ignited in a sersi-degenerate $0+N e+M g$ core. Whether subsequent evolution leads to a non-degenerate or a degenerate configuration deperds or neutronization during oxygen burning.

6) For $M>13 . M_{\odot}$, the star undergo non-kegererate burning to form an iron core.

Typical evolution for cases 4) - 6) will be discussed in $\$ 4$ - $̧ 6$, sespectively. (See Iben 1986 for smaller mass stars.)

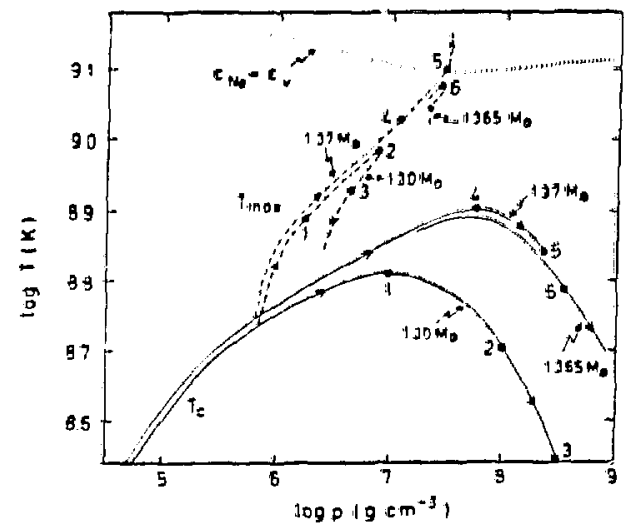

Fig. 1

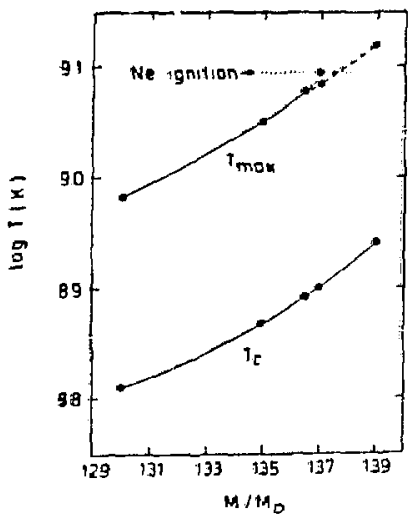

Fig. 3 1986).

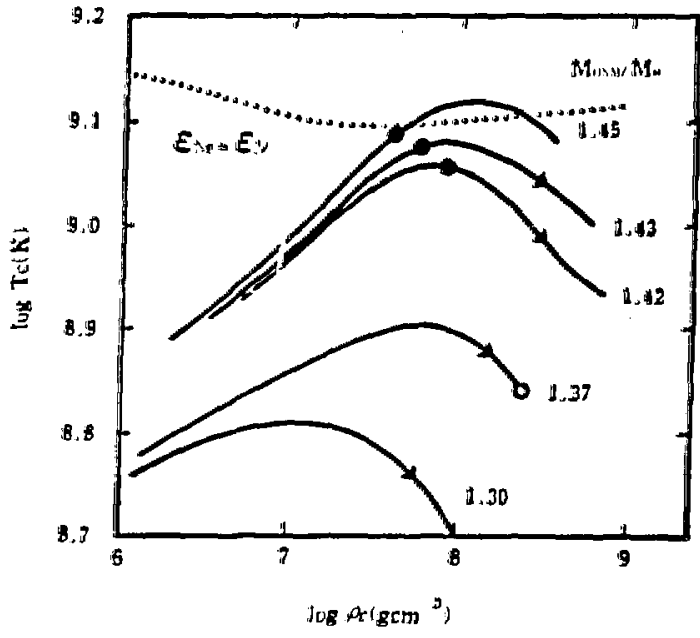

Fig. 2

Figure 1: Evolutionary change in the central density and temperature of reon stars during gravitational contraction (Nosnoto 1984a).

Figure 2: Sarne as Figure 1 but for neon stats of latger mass (Nomoto

Figure 3: The peak values of $T_{t e}$ and $T_{\text {tmsx }}$ given in Fugure $I$ as a funetion of neon star mass, M. The dotted line indicates the zeon ignition line (Nombto 1984a). 


\section{II. MODELS}

The present set of evolutionary calculations has been performed by Hashimoto and Nomoto (19s6) for helium stars of mass $M_{\alpha}=2.8 M_{\odot}, 3.0 M_{\odot}, 3.3 M_{\odot}, 0 M H_{\odot}$, and $3 M_{\odot}$. These helium star inasses corsespond to the main-seriwence mass of $\mathrm{Ar}=11,12,13,19$, and $25 \mathrm{Mr}$, respectively. The calculations started from the gravitational contriction of the helium stas. The input plysies is mostly the same 25 wsed in Nomoto (1984n) and Nomoto et al. (1954). The nuelent renetion network suas been developed by Hashimoto et al. (1983) and the reaction rntes are based on Fowler et al. (1975), Harris ct al. (1982), Caughla: et al. (1955), Woosing ct at $(1975,1979)$, and Thielemand (1980). The initial composition is

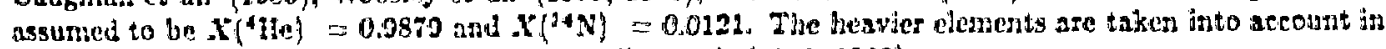
the opacity. The weak ates are taken from tivtler at al. (1980, 29B2).

In Figure 4, evolution of the central density, $\rho_{c}$, and tedperature, $T_{c}$, is shown for tikese cases. The approximate ignition lines for carbon, meon, oxygen, ard silicon, whitre the nuclear energy generation rate is equal to neintrino energy losses, are shoswin. The line for $\psi=10$ approximately divides the electron degenerate and non-degenerate scigos, where th is the chemical potential of an electron in units of $k T$. The lolium stars of $M_{a}=B$ and $C . S_{\odot}$ clearly undergo nuelear busning under non-degenerate conditions. For comparison, the evolutionary path of the $2.2 M_{\odot}$ helium core is shosw, which enters the strongly degenerate region after carbon burning. For $M H_{a}=2.8-3.3 \mathrm{M} \mathrm{F}_{\mathrm{O}}$, the $\mathrm{O}+\mathrm{Ne}+\mathrm{M} \mathrm{g}$ core becomes semi-degenerate and whether they will enter degenerate or non-derenerate Iegion is an interesting question. The evolution has been calculated up to the exhaustion of oxygen. The most important improvement from the previous calculations is that we include a more extensive nuclear reaction network and found tint even betore the oxygen exhaustion, the decrease in electron mole numis $:, Y_{e}$, is significant (see also Arnett and Thielemann 1985; Thielemann and Arrett 1085 ). The implication of these results is discussed for each case $534-50$.

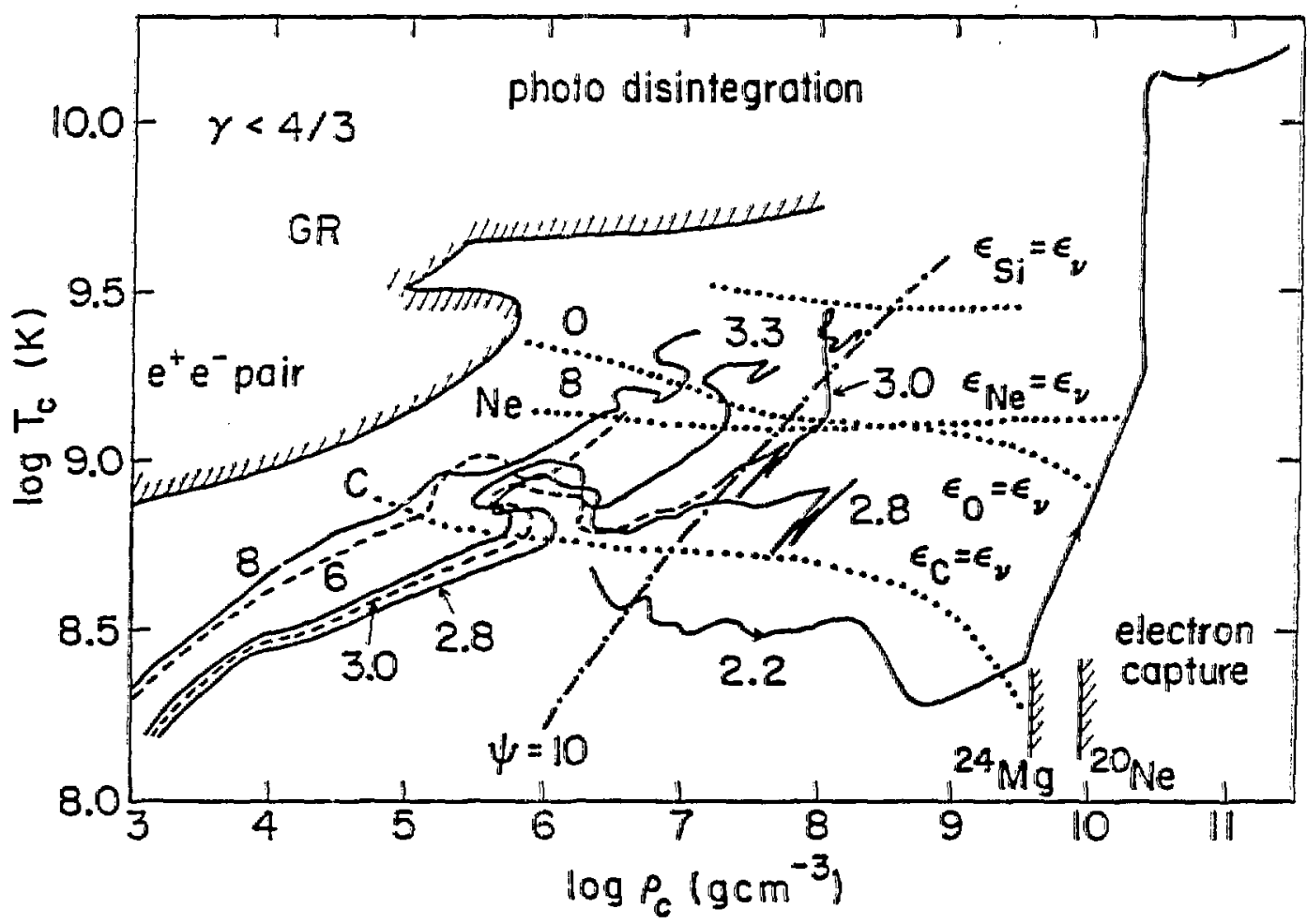

Figure 4: Evolution of the central density, $p_{e}$, and temperature, $T_{t c}$ for helium stars of muss $M_{a}=3,6$, $3.3,3.0,2.8$, and $2.2 .1 \mathrm{H}_{\odot}$. 


\section{EVOLUTION OF MASSIVE STARS $\left(M I \sim 25 M_{\odot}\right)$}

Stars with main-scquence mass of $M f_{\mathrm{mi}} \simeq 20-25 M H_{\odot}$ have been considered to be one of the most important sites of nucleosynthesis because they produce most species from oxygen to iron peak elements with reasonable accord to the solar abundance ratios (e.g., Woosley and Weaver 10B6b). It is also possible to compare the theoretcal prediction of uueleosyrnthesis with the observed ajundance of supernova ejecta which is obtained from supernova specta and supernowa semnants. Therefore it is important to calevlate the detailed nueleosynthesis history of stars in this mass range.

Reecnt revision of ${ }^{12} \mathrm{C}(\alpha, \gamma)^{10} \mathrm{O}$ rnte has substantinlly changed the evolution of nassive stars (ag., Fowlet 1084). Due to the $\sim 3$ times larger ${ }^{12} \mathrm{C}(\alpha, y)$ t0 $\mathrm{O}$ sate, the atowdance of carton producel by helivin burning is signifieantly smaller than the previous valut (Woosley and Weaver 1080, b). This affects not only the nueleosynthesis yicld, but also stellar structure and thus the find iron core mass. In the $25 \mathrm{Mr}_{0}$ model by Woosley and Weaver (1080A), the iron core is as anssive as 2.1 MO. The transition from the iton core mass from $1.35 M_{\odot}$ to $2 M_{\odot}$ occurs around $M_{\mathrm{m}} \sim 18 \mathrm{M}_{\odot}$. Such a latge iton core mass has given a big impact on supernova modeling because the prompt shock mechanistn does not work for such a core (c.g., Kaliana 1980; Hillebrandt 1986).

However, the carbon abundance is subject to uncertainties. It depends not only on ${ }^{22} \mathrm{C}(\alpha, 7){ }^{16} \mathrm{O}$ rate, but also on how the convective core grows, and thus, on the treatment of the convertive core edge and overshooting. In particular, if the convection core grows substantially near the end of helium burning, fresh helium is rzixed into the high temperature region and processes ${ }^{12} \mathrm{C}$ jnto ${ }^{16} \mathrm{O}$ very efficiently. Since so reliable theory cf convection, especially non-local theory, exists at the moment, it is necessary to explore the various possible evolutionary paths within a uncertain sange. As a fisst step, we report our prelininary calculations for the helium star of $M_{a}=8 M_{\odot}$ which is almost equivalent to $M_{I_{m}}=25 M_{\odot}$. We adopt the Schwarzshild criterion for convective stability and neglect the overshooting (e.g., Langer 1986).

\subsection{Stellar Structure}

First let us look at some evolutionary features of this star from the density distribution in Figure 10 . The stage numbers sefer to 1: helium burning, 2: exhaustion of helium, 3: carbon ignition, 4: neon ignition, 5: oxygen ignition, and 6: exhaustion of oxygen. The evolutionary change in the density distribution depends on the timescale of entropy loss from the central segion, which is mainly determined by the neutrino luminosity, $L_{\nu}$. During the quasi-static contraction, the decrease in entropy due to reutrino losses promotes the contraction as seen from Eq.(3). For nuclear burning stages, nuclear luminosity, $L_{n}$, is bolanced witli $L_{\nu}$ so that the entropy in the convective layer is almost stationary. However, the depletion timescale of suclear fuel is determined by $L_{n}$ and thus related to $L_{\nu}$. As $T_{c}$ increases, $L_{\nu}$ increases sapidly, which accelerates the evolution. As seen from Figure 10, the whole helium stat contracts up to stage 2. Later, the density structure in the outer layers is practically frozen since the timescale of energy trassport from the central region to the nuter layers is much longer than the evolutionary timescale near the center. In this sense, the effective core mass, $M_{\text {core, }}$ which seplaces $M$ in $E q .(1\}-\{3)$, is decreasing. When shell busring is active, its location, $M_{r}$, gives the approximate value of $M_{\text {core }}$ as discussed in $\$ 2$.

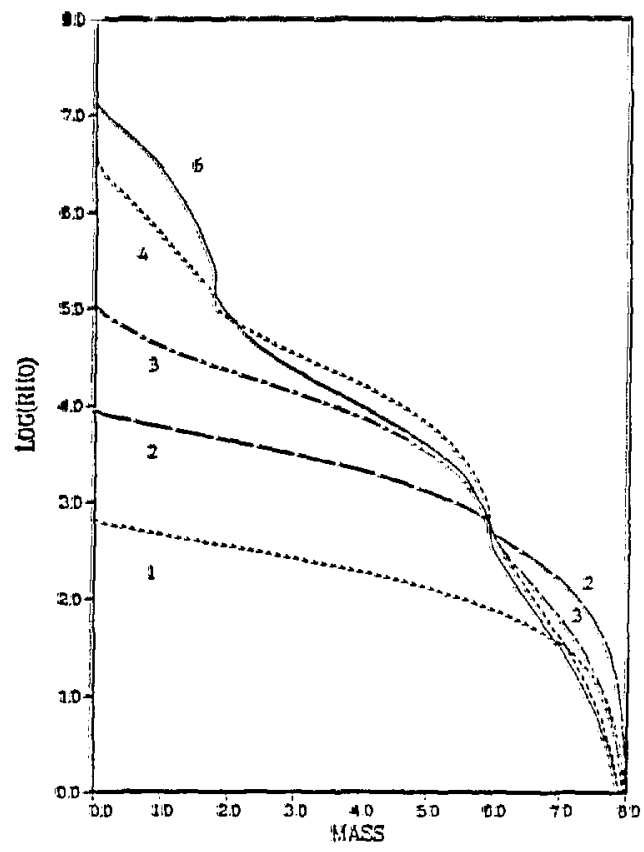

Figure 5: The evolutionary change in the density distribution for a helium star of $M_{a}=8 \mathrm{M}$. 
4.2 Helium, Carbon, asd Neon Burning and Carbon Aburdance

The helium burning stage lasts for $7 . .15 \times 10^{5} \mathrm{yz}$ and a $\mathrm{C}+\mathrm{O}$ core of $6.0 \mathrm{M} \odot$ forms. "The Inass fractions of carbon and oxygen are $X\left({ }^{12} \mathrm{C}\right)=0.19$ and $X\left({ }^{16} \mathrm{O}\right)=0.79$, respectively, as seen in Figure 6 . This amount of crebon is small due to the faster ${ }^{12} \mathrm{C}(\alpha, \gamma)^{10} \mathrm{O}$ acaction, but still sufficiently jarge that the carbon burning rate exceeds the neutrino cnergy losses when $T_{i c}=8.0 \times 20^{8} \mathrm{~K}$. Carbon burning starts in the center (stage 3: $8.4 \times 10^{3}$ yr aftcr exhiustion of helium) and forms a convective core of $0.27 M_{0}$. The compostion structure at carbon igntition is shown da Figure 6 . The carben bnrnisig plase lasts only $100 \mathrm{yr}$. When carbon is altuost depleted in the core, the restitant composition is $X\left({ }^{10} 0\right)=0.70$ and $X\left({ }^{20}\right.$ Ne) $=$ 0.25. Because of the suall earbon abundance, the $\theta /$ Ne satio is large compared with the model based on the old low ${ }^{12} \mathrm{C}(\alpha, \gamma){ }^{16} \mathrm{O}$ ante (Weaver ct al. 1075). The carbon burtitig layer shifts to the rater shell and a convective shell develops at $M F_{r}=0.94-1.73 . M_{0}$. When neon is ignited in the center (stage 40 yr after exhaustion of carbon), only a trace of carbon is left interior to $M f_{r}<1.78 M_{0}$, and a coswective carbon burning shell forms at $M I_{r}=1.79-5.4 . L_{0}$. Neon burritig lasts for $0.4 \mathrm{y}$ : asjd forms a convective core of $0.42 W_{\odot}$. After exhaustion of neon th the center, an oxygen-rich core of $X\left({ }^{16} \mathrm{O}\right)=0.82$ and $X\left({ }^{24} \mathrm{Mg}\right)=$ 0.08 forms (Figure 8 ) and a convective neon burning stell appears at $M_{r}=0.42-0.90 \mathrm{M} \mathrm{C}_{\mathrm{H}}$.

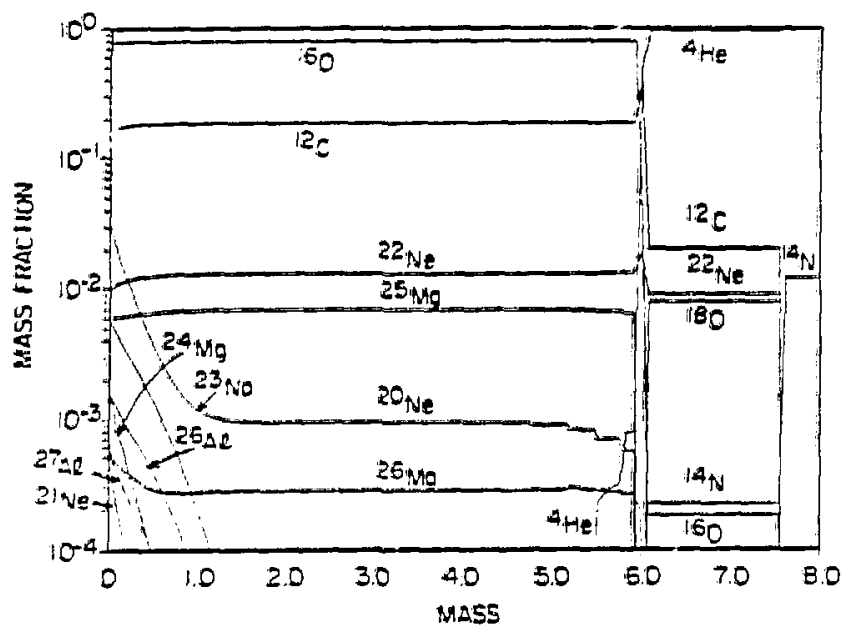

Figure 6: Composition of a heliusen star of $M_{a}=8 M_{0}$ at carbon ignition (stage 3).

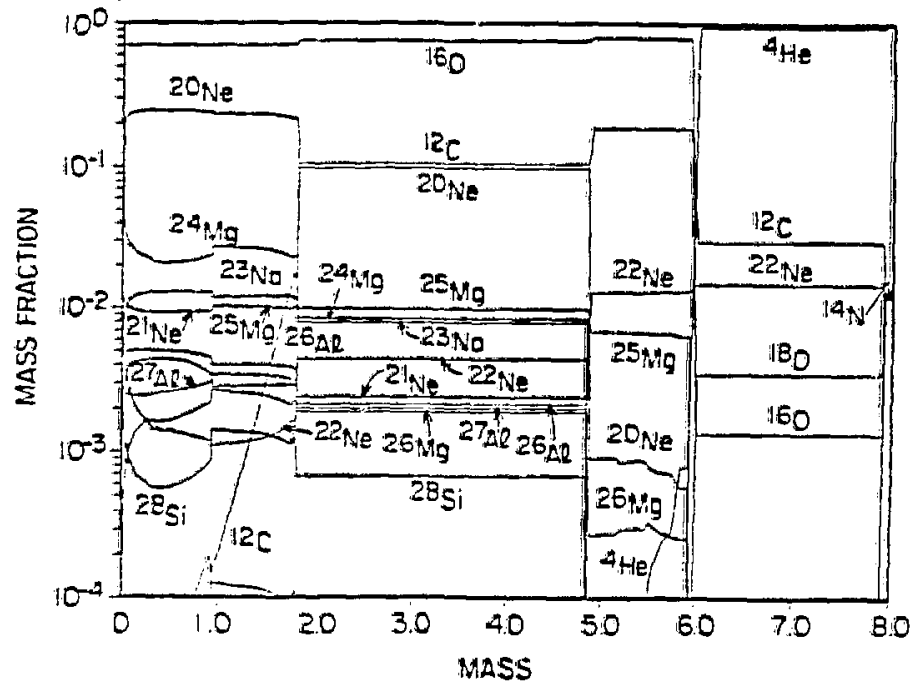

Figure 7: Composition of a helium star of $M_{a}=8 M_{\odot}$ at ncon igtition (stage 4), 


\subsection{Oxygen Burning Stage}

At stage $E$, oxygen is ignited in the center (Figure 8 ). A convective core extends to $\mathrm{M}_{\mathrm{r}}=0.99 \mathrm{M} 0$. Oxygen burning synthesices silicon and sulfur - zich elements. As oxygen burning procects, the produet is getting

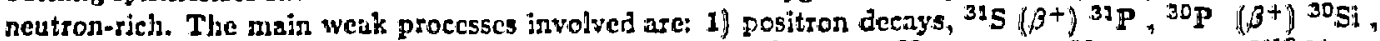
and ${ }^{34} \mathrm{Cl}\left(\beta^{++}\right)^{34} \mathrm{~S}$ and 2) election captures, ${ }^{33} \mathrm{~S}\left(\mathrm{e}^{-}, \nu\right){ }^{33 \mathrm{P}}$ and ${ }^{35} \mathrm{Cl}\left(\mathrm{e}^{-}, \nu\right)^{35} \mathrm{~S}$. When $\mathrm{X}\left({ }^{16} \mathrm{O}\right) \approx$ $0.013, Y_{\text {e }}$ drops to 0.493 (neutron excess $n \equiv 1-2 Y_{0}=0.014$ ) in the convective core whete ${ }^{28} \mathrm{Si}, 32 \mathrm{~S}$, and ${ }^{38}$ Ar are the main constituents (Figure 9 ). At this stage, a convective shell of neon burning starts to form at $M f_{r} \geq 1.00 M \rho_{0}$.

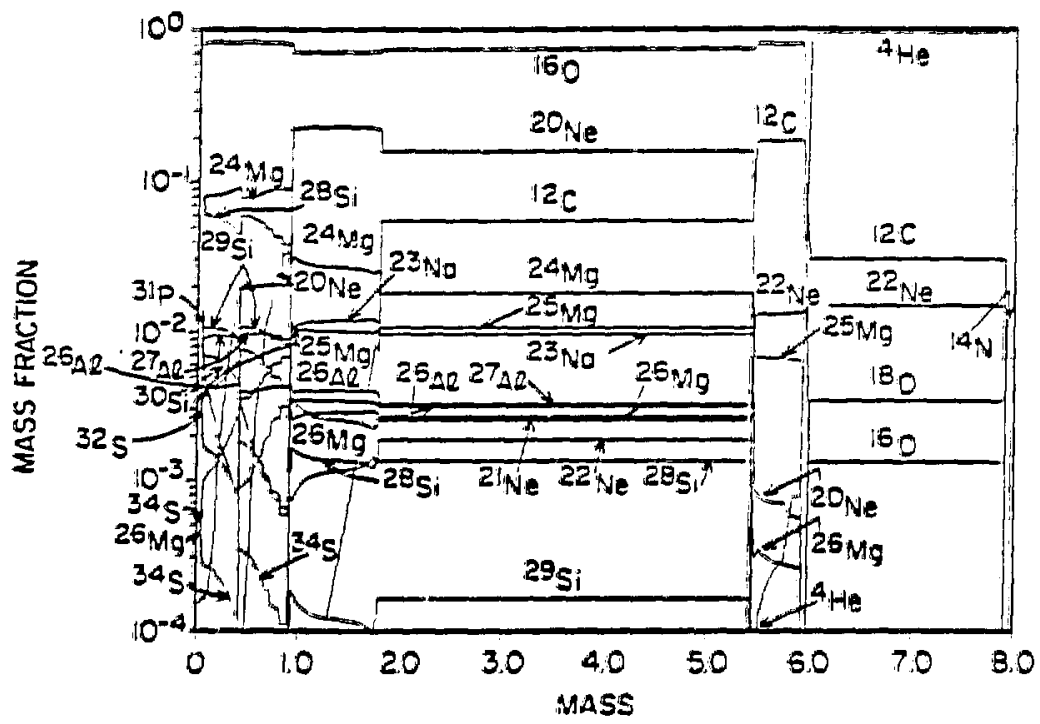

Figure 8: Composition of a helium star of $M_{a x}=8 M_{\odot}$ at oxygen ignition (stage 5).

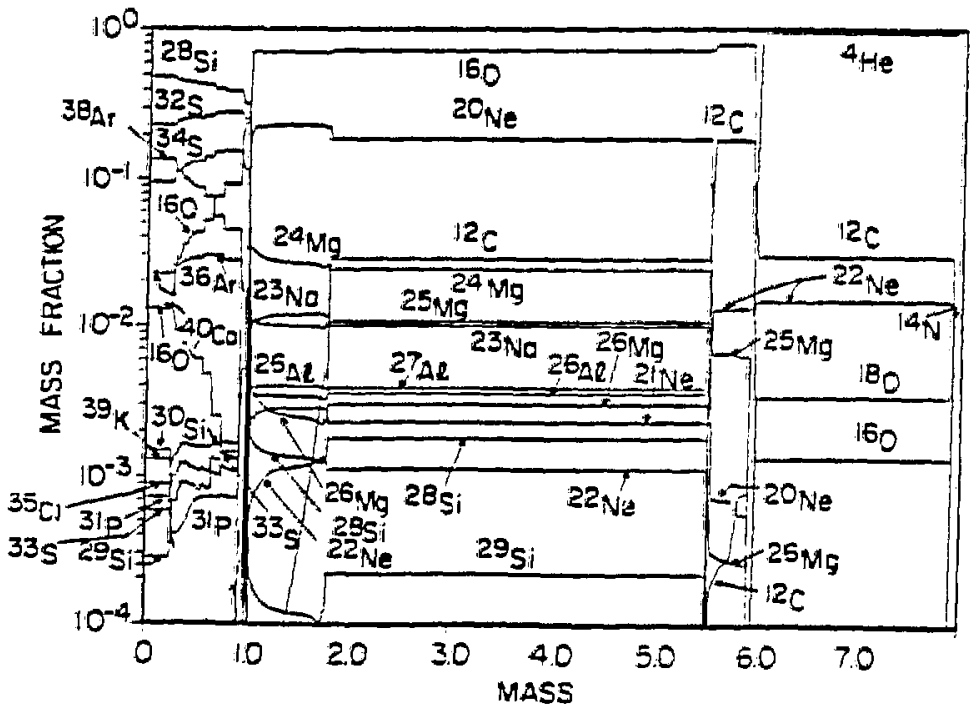

Figure 9: Composition of a helium star of $M_{\alpha}=8 M_{\odot}$ gear the end of ox gen burning where $X\left({ }^{16} O\right)=$ 0.013 [stage 6). 


\subsection{Comparison witin the Previous Models}

Our result is similar to the $25 M_{0}$ model of Woosie; and Weaver (1986a; WW25 model) in the scnse that the faster ${ }^{12} \mathrm{C}(\alpha, \gamma)^{10} \mathrm{O}$ reaction reduces the abundances of carbon, theon, and magresium, while at increases the oxygen abundance. On the other hind, there are some differences. In the WW25 motiel, no convective core appears for earbon and neon burniss phases, while, in ous model, both carbon and neon burwing dominate the nestrino energy losses and thus, a small convective core and then an extensive convective sizell form, In particular, convective carbon burning shell cxtcnding from $M_{\mathrm{r}} \equiv 1.79 M_{0}$ to 5.4 $M_{\odot}$ is quite active during neon and oxygen buming. As a restalt, a sharp entropy increase appears at $M_{P} \equiv$ $1.8 \mathrm{M}_{\odot}(s=5.0 \mathrm{k} / \mathrm{H}$ in the carbon burning hell). The esistence of active burning shell anakes the eftective corc mass in Eq.(1) smaller, which correspon is to the smaller central motropy. In fact, at oxygess ignitiots, $s_{c}$ is as low as $1.9 \mathrm{k} / \mathrm{H}\left(\psi_{\mathrm{e}} \approx 2.8\right)$ which is aloust equal to that of the $15 \mathrm{M} \%$ W W wodel ard Bualles than that of the WW25 model by a factor of 2.5. The entropy distribution in our model is very sitsilar to the old $25 M_{\odot}$ model by Weaver, Woosley, and Fuller (1085, WWF). This is because our carbon abundarce is about twice that of the WW model, which may be due to differences in the treatment of convection. Since the WWF $25 M_{\odot}$ zodel foruns a $1.35 M_{\odot}$ iron core, our model is expected to form sueh a small iron core.

During oxygen burning, positron emissions and electron captures increase 7 . Tuough $n \simeq 0.014$ at $X\left({ }^{16}(0)\right.$ $\simeq 0.013$ in this model, $\eta$ is larger in smaller mass stars and has important effects on the later evolution and תucleosynthesis (65). Nucleosynthesis in our model is consistent with more detailed network calculation based on the polytropic models by Thielemann and Arnett (19B5).

\section{EVOLUTION OF $10-13 M_{\odot}$ STARS}

The evolution of stars in the mass range of $M_{m}=10-13 M_{\odot}\left(M_{a}=2.5-3.3 M_{\odot}\right)$ is significatitly different from $25 M_{\odot}$ stars, being more complicated and sensitive to the steliar mass. This is because electrons become somewhat degenerate, which affects the structure of the core. Stars its this inass tatige undergo non-degenerate carbon burning and form an oxygen-neon core whose mass, MoNe, is in the tange of $1.37-1.5 \mathrm{M}_{\odot}$. The core mass HONe $_{\mathrm{O}}$ is large enough to ignite then, yet the core is semi-degenerate and the degree of degeneracy depends sensitively on how close $M_{O N e}$ is to the Chandrasekhar toass.

\subsection{Nucleosynthesis during Helium and Carbon Burning}

The evolution during helium and carbon burning is qualitatively simillar to the $25 \mathrm{MF}_{\odot}$ star but quantitatively different due to the smaller mass and thus lower central temperature during nucuear burning. As an example of this mass range, Figures $10-12$ show the chemical composition of the helium star of $M_{\alpha}=2.8 M_{\odot}$ $\left(M_{\mathrm{ms}} \simeq 11 \mathrm{M}_{\odot}\right)$. Helium burning forms a $\mathrm{C}+\mathrm{O}$ core of $1.23 \mathrm{M} \odot$. The abundances are $\mathbf{X}\left({ }^{12} \mathrm{C}\right)=0.20$ and $X\left({ }^{16} \mathrm{O}\right)=0.69$ (Figure 10 ). The mass fraction of carbon is $0.28-0.29$ for this mass arage, which is larger than in the $25 M_{0}$ motel due to the lower central temperature. The old ${ }^{12} \mathrm{C}(\alpha, \gamma){ }^{10} \mathrm{O}$ rate gives $X\left({ }^{12} \mathrm{C}\right) \simeq 0.5$ for $M_{\mathrm{ms}} \sim 10 M_{\odot}$ (e.g., Arnett 1972). The helium burning shell advarces gradually in waiss and thus the $\mathrm{C}+\mathrm{O}$ core mass grows as $M_{C O}=1.345,1.415$, and $1.423 M_{\odot}$ at the stage of carbon jgnition: exhaustion of carbon, and reon ignition, respectively. Note that $M_{C O}$ is less thar the Chardrasekhar mass but larger than the critical mass of $1.37 M_{\odot}$ for neon jgnition.

Carbon burning ignites in the center and forms an oxygen-neon core of about $0.6 M_{\odot}$. The oxygen-neon core contains more oxygen than neon and their mass fractions are $X\left({ }^{16} \mathbf{O}\right)=0.56, X\left({ }^{20} \mathrm{Ne}\right)=0.33$, and $\left.X{ }^{24} \mathrm{Mg}\right)=0.06$ (Figure 11 ). The oxygen/neon ratio is almost the severse of that in the old models because of the small carbon abundance in the present model. The ${ }^{24} \mathrm{Mg}$ abundance is also small for the same reason. 


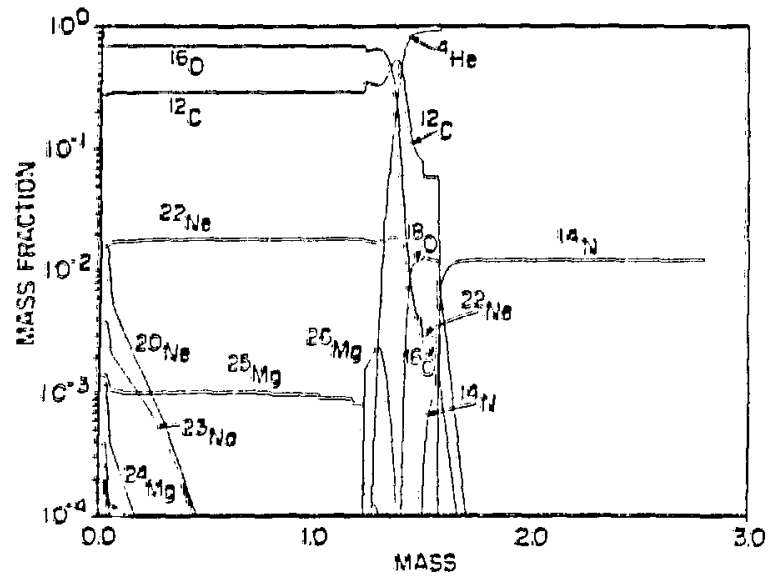

Figure 10: Abundances in the $2.8 M M_{0}$ heljun star at carbon iguition in the center (stage 2 ).

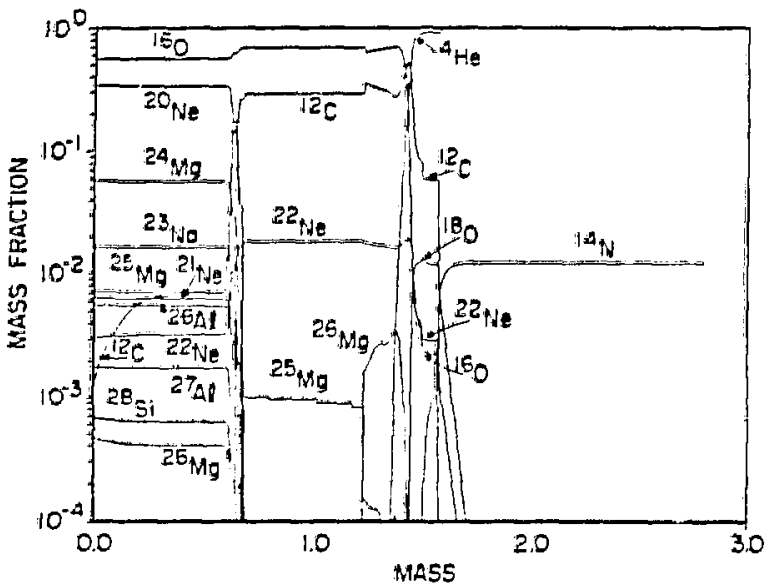

Figure 11: Same as Figure 10 but at exhausion of carbon (stage 3).

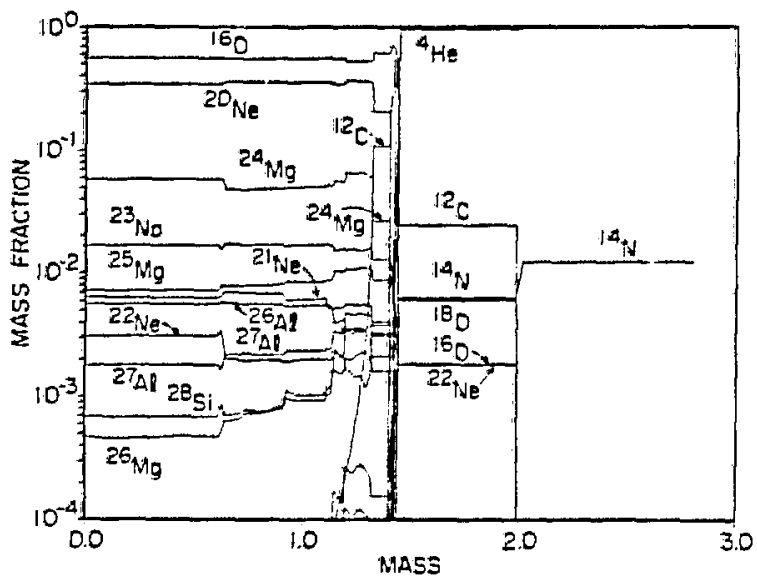

Figure 12: Same as Figure 10 but at neon ignition (stage 6). 


\subsection{Semi-Degenerate Oxygen-Neon Core and Off-Center Neon Ignition.}

The oxygen-neon core grows and contracts as the carbon burning shell advances. The evolution of $\left(p_{c}, T_{c}\right)$ in Figure 3 is determined by the increasing oxygen-neon core mass, MoNe as given in Eq. (1). Since the core is somewhat degenerate, compressional heating is slower than the neutrino cooling. Therefore, the central temperature does not increase while $\rho_{\mathrm{c}}$ increases. The electrons become degenerate in the central region, i.e., the $\left(\rho_{c}, T_{c}\right)$ path enters the region of $\psi>10$. When $M_{\text {ONe }}$ reaches the critical mass of $1.37 M_{\odot}$, neon burning ignites not in the center, but in the outer shell. The location of the neon ignition depends on the degree of election degeneracy and thus on the stellar mass.

The formation of a semi-degenerate core gives several distinct features to stars of $A I_{a}=2.5-3.2 M M_{\odot}$. Let us first look at the overall evolution from the evolutionary elange in density and temperature distribulions of the helium star of $M_{a}=2.8 \mathrm{M}_{\odot}\left(\mathrm{M}_{\mathrm{tn}} \sim 11 \mathrm{M} O\right)$ (Figures 13 and 14). The stage numbers correspond to 1: exhaustion of helium, 2: ignition of carbon buming, 3: exhaustion of carbon, 4-5: development of a temperature inversion in the O-Ne core, 6: off-eenter ignition of neon, 7-B: propagation of the neon burning layer.

First we note that the star forms a nuite remarikable core - envelope structure (Figure 10). As the core contracts, the helium envelope expandis. The helium burning shell is a node and a very steep density gradient appears at the helium burning shell $\left(M_{r}=1.42 M_{\odot}\right)$. The zadius of this helium star Ieaches 12 $R_{\odot}$. This is the same phenomena as a star expands from the main-sequence to the giant branch (Sugimoto and Nomoto 1980,. For $M_{\alpha}=8 M_{\odot}$, the core - envelope feature is less pronounced, because the evolutionary timescale after carbon burning is too short for the effect of core contraction to be transmitted to the helium layer.
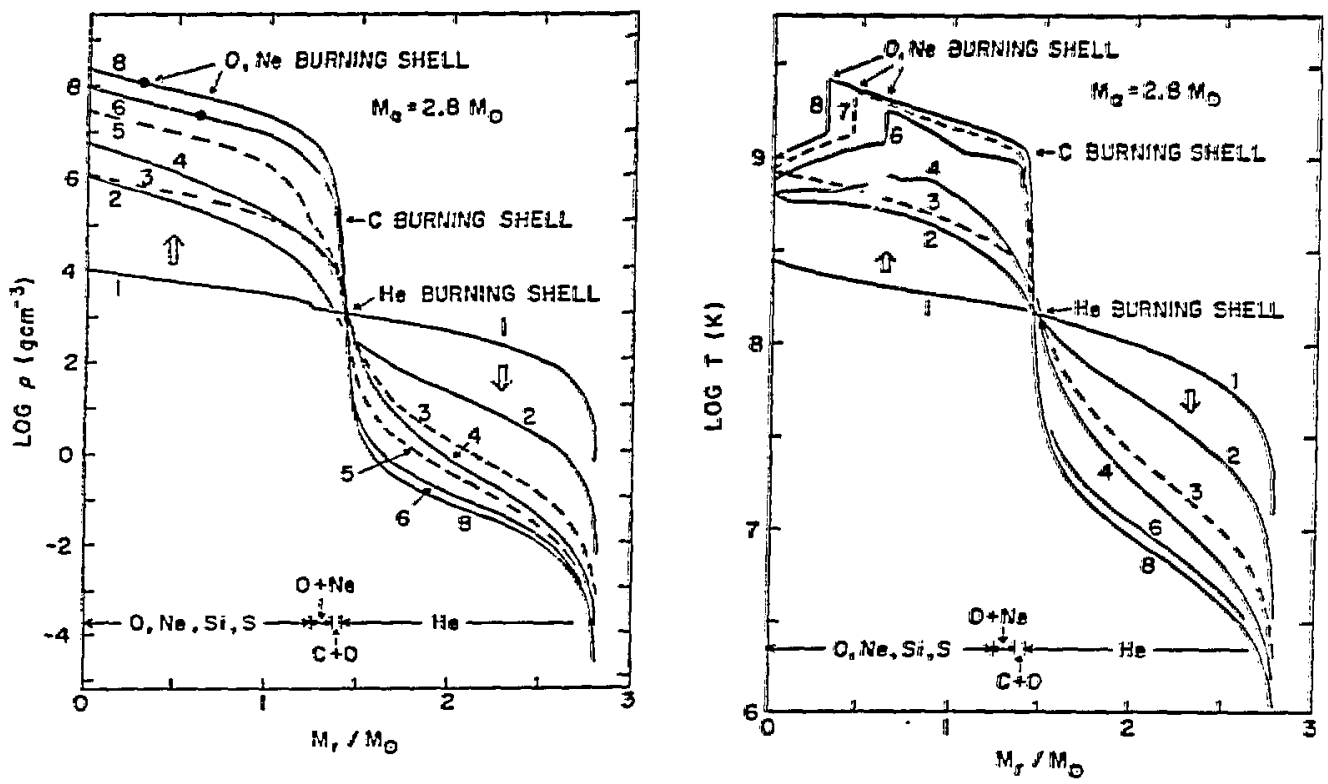

Figure 13 (left): Evolutionary change in the density distribution for $M_{a}=2.8 \mathrm{M} \odot$.

Figure 14 (right): Same as Figure 13 but for termperature. 
Secondly, stars in this range develop a temperature inversion in the central region (Figure 11), because neutrino energy losses are faster at higher densities. In such a core. the pressure necessary to sustain hydrostatic equilibrium is provided by degenerate electrons. The temperature inversion leads to an offcenter ignition of neon at $M_{r}=0.78,0.64,0.30$, and $0.10 M_{\odot}$ for $M_{\alpha}=2.6,2.8,3.0$, and $3.2 M_{O}$, respectively (see, e.g., Nomoto $1984 a$; Habets 1985,1986 ). For $M_{\alpha}=3.3 M_{0}$, electron degeneracy is so wenk that the temperature inversion does not appear and neon ignites at the center. Therefore, the critical main-sequence mbss that discriminates beween off-center and central ignition of neon is $M_{m a} \sim 13 M_{\odot}$.

\subsection{Propagation of Neon-Oxygen Buraing Layer}

When ncon ignites off-center, shell burning is unstable to a fish due to election degeneracy. The Sash incresses tine temperature to as high as $2 \times 10^{9} \mathrm{~K}$ and forms a sharp temperature jump as seen in Figure 14. Subsequently the neon burning shell propagates inward (Figure 14). An important question is whether or not the burning shell reaches the center. If not, a degenerate oxygen-neon core is left unburned as found by Barkat et al. (1974) and also in the Woosiey and Weaver's (1986a) $11 M_{0}$ model. This is crucial in determining the final fate of stars.

Figure 15 shows the propagation of the neon burning layer for $M_{a}=3.0 M_{\odot}$. As the burning front moves to the higher density layers, the temperature increases to $2.0-2.5 \times 10^{\circ} \mathrm{K}$. At such high temperature, oxygen also burns to synthesize silicon and sulfur. The composition profle during the propagation is shown in Figure 16. The burning front propagates all the way to the center. Since the density at the neon burning front does not become so high $\left(\rho<10^{5} \mathrm{~g} \mathrm{~cm}^{-3}\right)$ and also neon ignites layer by layer, the released energy in O-Ne flash is too small to induce major dynamical effects.

For slightly smaller mass of $M_{a}=2.8 M_{0}$, on the other hand, the neon burning front reaches dentities as high as $\rho>10^{\circ} \mathrm{B} \mathrm{cm}^{-3}$. Then the neon shell Hashes become so explosive that a dynamical event, such as ejection of a helium layer, is expected (also Woosley et al. 1980). Therefore, it is crucial whether the neon burning front can reach high densities or is quenched by neutrino cooling at an earlier stage.

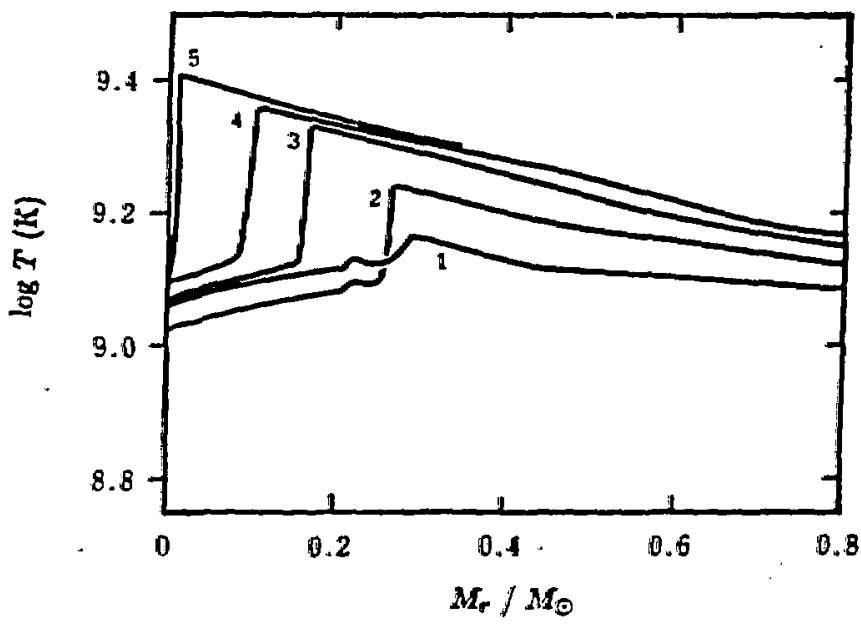

Figure 15: Change in the temperature profile during the propagation of the neon burning front for $M_{\alpha}=$ $3.0 M_{\odot}$. 
The propagation mode of neon burning front is not hent conduction but compressional heating due to gravitational contraction of the oxygen-neon core. This can be seen from the increase in density after the reon ignition in Figure 13. For $M_{a}=3.0 M_{\odot}$, it takes only 2 yr for the burning front to propagate to the center. This is much faster than conduction (also Ikeuchi et al. 1972; Woosley et al. 1980). In ordes to sustain the propagating front, compressional heating should dominate neutrino cooling. In other words, the core mass interior to the helium burning shell should be sutficiently large to keep $c_{5}<0$ until $T_{c}$ reaches the neon ignition temperature. If the corn mass is too small relative to the Chandrasekhar mass, electron degeneracy changes the sign of gravothenal specific heat of the core and then the temperature starts to decrease.

In ordet to clarify this point, simple oxygen-neon star models have been calculated with neon burning artificially suppressed as already shown in Figure 2 . Here the stare of mass $M$ owe are undergoing gravitational contraction. The result shows that for $1.46 M_{\odot}>M_{O N e}>1.37 M_{\odot}$ the neon ignition temperature is reached first in the outes shell at the stage marked by open cicle in Figure 2. Afterwards the central temperature also reaches the neon ignition line for $M_{O} N_{e}>1.44 M_{O}$, while $T_{c}$ starts to decrease without igniting neon for smaller mass stars.

If we apply this criterion to the case $r M_{a}=2.8 M_{\odot}$, its core reass is onily $M_{O N e}=1.42 M_{\odot}$ so that neon burning will be quenched with some unburned oxygen-neon in the central region. However, the above criterion is obtained for $Y_{t}=0.5$. In the actual seon - oxygen burning, $Y_{0}$ is decreasing due primerily to electron capture on ${ }^{33} \mathrm{~S}$ and ${ }^{35} \mathrm{Cl}$. For $M_{\alpha}=2.8 \mathrm{M}_{\odot}$, the density at the burning front is as high as $10^{8}$ $\mathrm{g} \mathrm{\textrm {cm } ^ { - 3 }}$ so that $Y_{e}$ is as low as 0.48 due to electron captures. Such a small $Y_{e}$ implies that the core mass exceeds the Chandrasekhar mass that is now $\sim 1.38 M_{\odot}$ and thus the gravothermal specific heat of the core remains negative. Thercfore, the central temperature continues to inerease up to the neon ignition temperature. Then the harning layer will reach the center. The propagation of the oxygen-neon shell burning is self-sustanined because electron captures in the burning layer promotes the contraction. This implies that the density at the burning front will become high enough to induce explosive reon fiashes for $M_{\mathrm{a}}=2.6-2.8 M_{\odot}$. It may or znay not lead to the ejecton of a helium layer (Woostey et al, 1980). The quenching of neon burning would be the case only for very limited mass range, if it occurs at all. The use of a sufficiently large nuclear reaction netiwork is crucial for the study of this mass range.

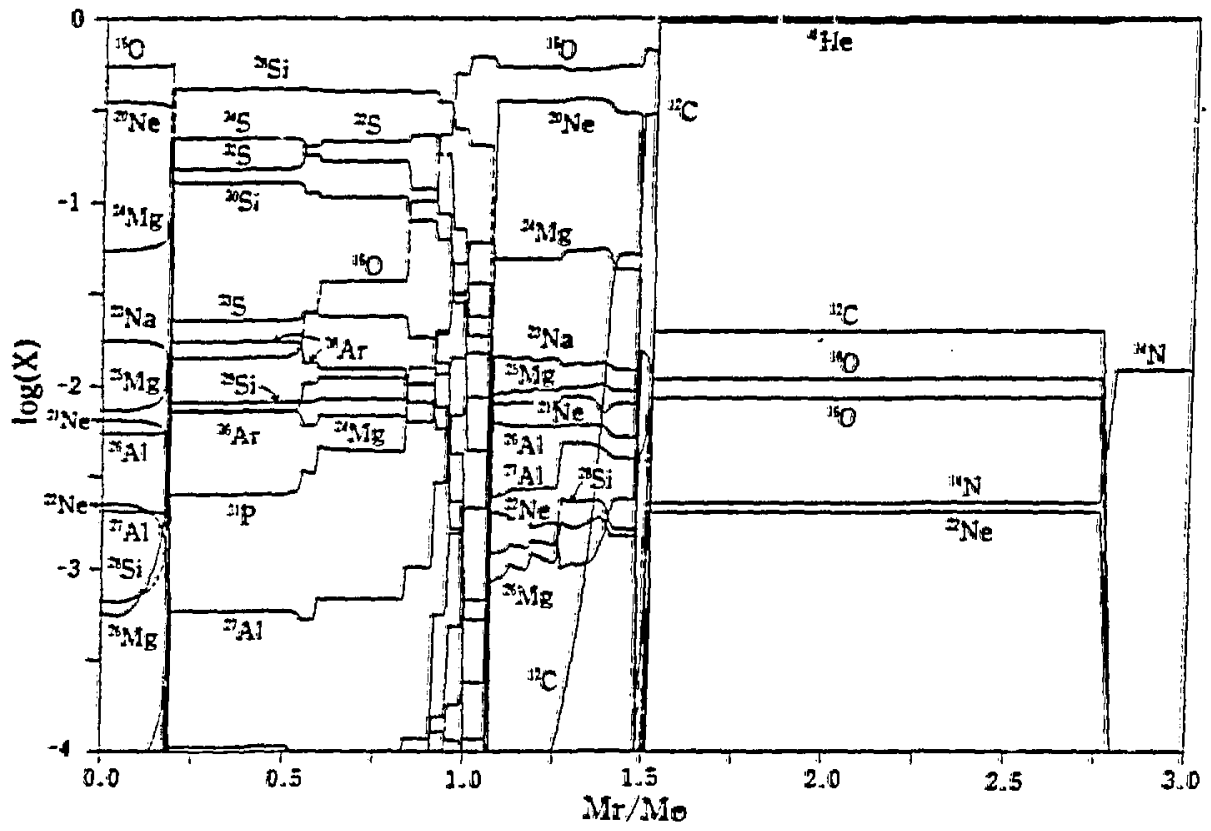

Figure 16: Composition of the helium star of $M_{\alpha}=3.0 M_{\odot}$ during the propagation of geon and oxygen burning front. 


\section{EVOLUTION OF 8 - 10 M\% STARS}

For this mass range, electron degeneracy becomes significant already in a $\mathrm{C}+\mathbf{O}$ core. Therefore, several features of the semi-degenerate core discussed in $\$ 5$ appear earlier, i.e., before carbon ignition. We describe the evolution of a helium core of $M_{a}=2.2 M_{\odot}$ as a typiral example for this mass range (Nomoto 1984b). (This is not a helium star but a core embedded in the hydrogen-rich envelope of a star with $M_{\text {ms }}=\mathrm{B} . \mathrm{B}$ $\left.M_{O}\right)$ The evolutionary path of $\left(\rho_{c}, T_{c}\right)$ is seen in Figure 4 . Chemical evolution of the core through the eni of the dredge-up of aelium layer is shown in Figure 17. Evolutionary changes in the photon luminosity, $L_{p h}, L_{n}$, and $L_{\nu}$ are shown in Figure 18. Figure 19 shows elianges in $p_{c}, T_{e}$ and $\psi_{c}$. For the stages where the teinperature inversion appears, the maximum temperature within the core, $T_{\text {max, }}$ is also plotted. This calculation used the old ${ }^{12} \mathrm{C}(\alpha, \gamma){ }^{16} \mathrm{O}$ sate so that the cetnily of eiemental abundances are not discussed here.

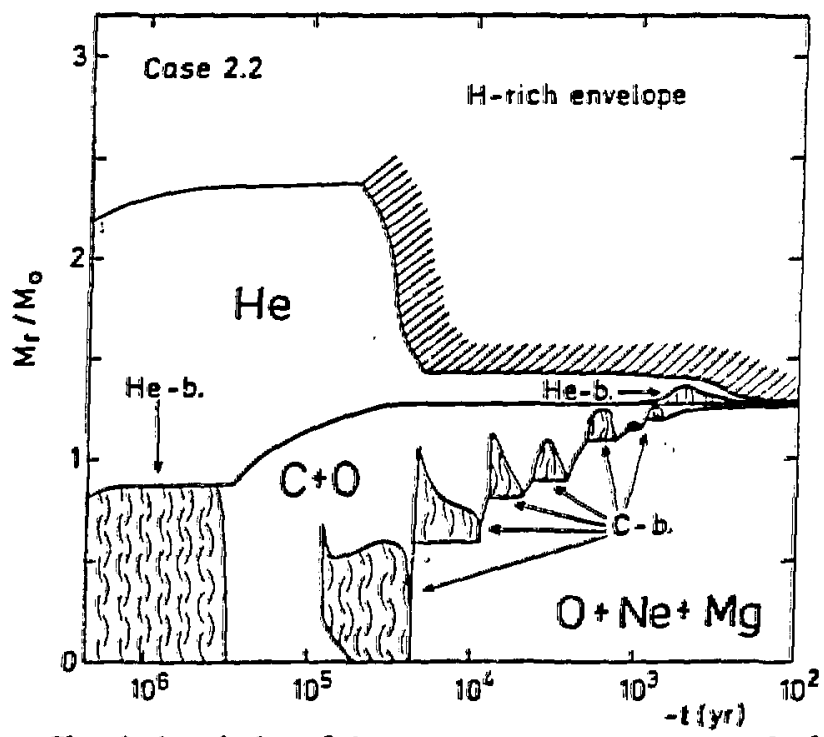

Figure 17: Chemical evolution of the core through the end of the dredge-up of helium layer.

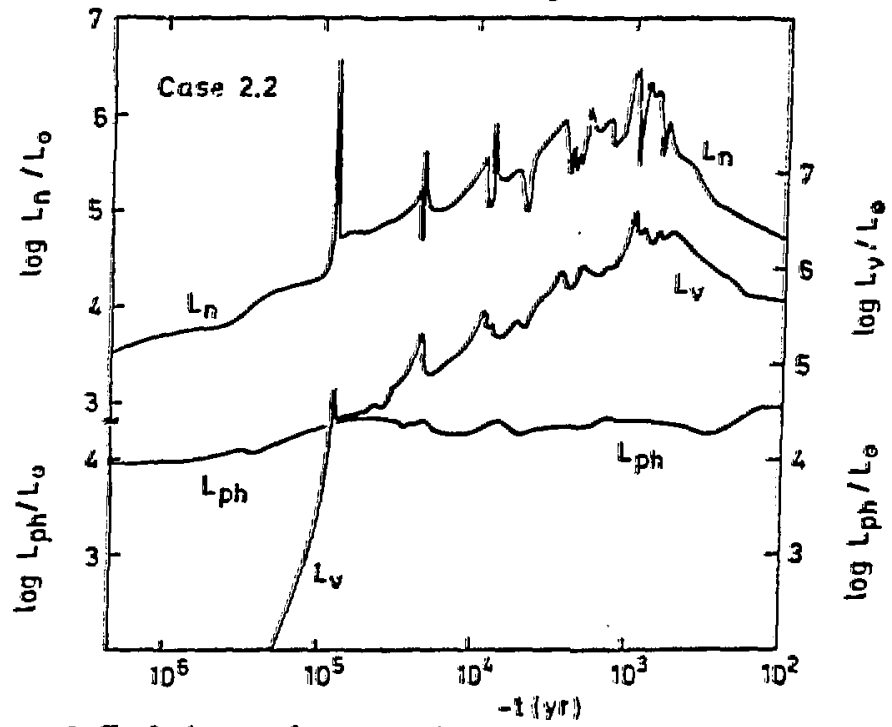

Figure 18: Evolutionary changes in the photon Juminosity, $L_{\mathrm{ph}}, J_{ \pm n}$, and $L_{\nu}$ 


\subsection{Carbon Burning}

In a semi-degenerate $\mathrm{C}+\mathrm{O}$ core, a temperature inversion appears due to geutrino cooling. This leads to off-center ignition of carbon when the $\mathrm{C}+\mathrm{O}$ core mass exceeds the critical mass of $1.06 \mathrm{M}_{0}$. (For $\mathrm{M}_{0}>$ $2.6 M_{\odot}$, carbon ignites in the center.) The off-center carbon burning shell moves inward all the way to the center as neon shell burning does for $M_{\alpha}=2.8-3.2 M_{\odot}$ (Figure 17). However, the propagation mode is different. For neon burning, it is compressional henting. On the other hend, it is heat conduction for carbon burning. Once ignited, there is no way to provest the burning front from reaching the center as shown for acercting $\mathrm{C}+\mathrm{O}$ white dwar's (Saio and Nomoto 1985; Woosley and Weaver 1980a).

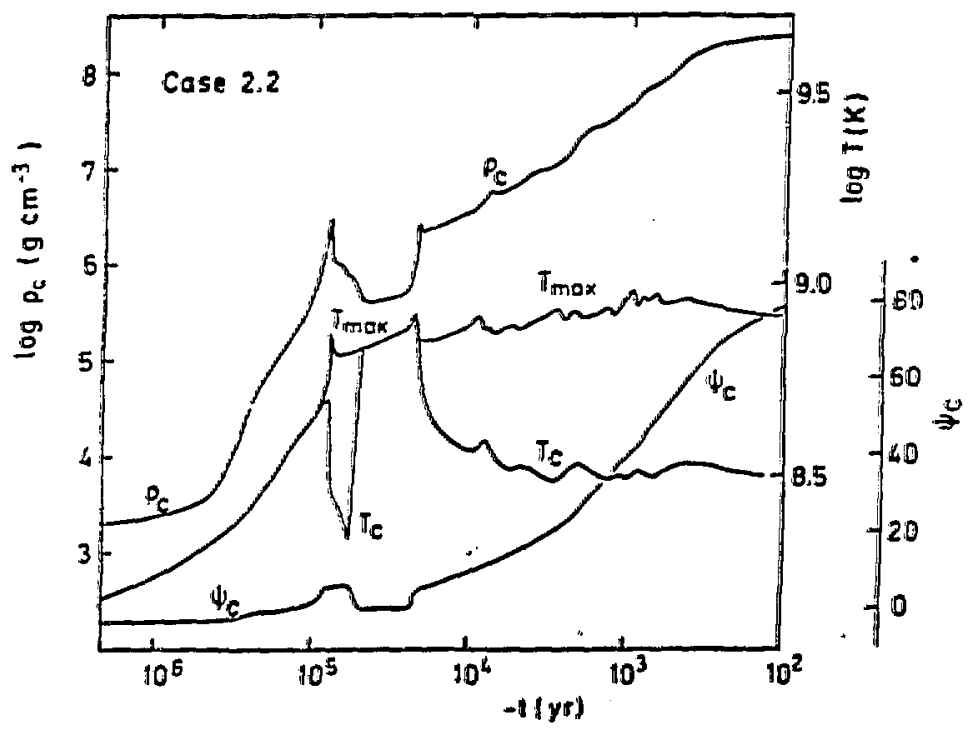

Figure 19: Changes in $p_{c}, T_{c}$, and electron chemical potential, $\psi_{s}$, in units of $k T$.

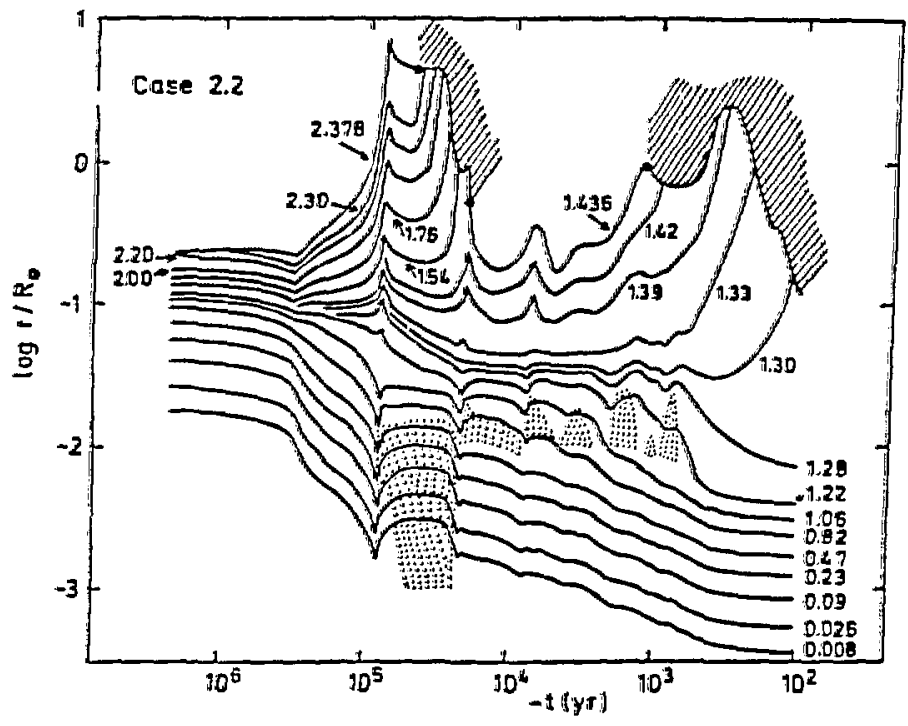

Figure 20: Evolutionary cinanges in the radial distances from the center for several Lagrangian shells indicated by $M_{r} / M_{\odot}$. 


\subsection{Formation of Strongly Degenerate O-Ne-Mg Core}

After exhaustion of carbon in the central region, an $\mathrm{O}+\mathrm{Ne}+\mathrm{Mg}$ core forms. Its mass increases through irregular carbon shell burning. Figure 20 shows evolutionary changes in the radial distances of Lagrangian shells from the center. Here the dotted regions are convective due to carbon bu:.ring. It is seen that the carbon burning sheli is almost stationary around $r \simeq 0.01 R_{\odot}$. The core materials contract, being heated up to the carbon ignition temperature and moving through the burning shell after being converted into oxygen-neon. In this way, $\rho_{e}$ increases as the mass of the oxygen-neon core increases. At the same time, $T_{\varepsilon}$ decreases down to $3 \times 10^{\mathrm{g}} \mathrm{K}$ due to neutrino emission. It is clear that the specific heat of the core is now positive. This is the important difference from more rnassive stars. Accordingly, the core becomes strongly degenerate as seen in Figure 4.

Since the advance of the oxygen-neon burning shell stops near the helium burning shell at $M_{+}=1.2 B M_{\odot}$, the oxygen-neon core mass does not exceed the critical mass of $1.37 M_{\odot}$ so that neon in never ignited. The Inaximum temperature attained is $9 \times 10^{3} \mathrm{~K}$ which is far below the igrition temperature of neor. This is the case for $M_{\mathrm{a}} \approx 2.0-2.5 M_{\odot}\left(M_{\mathrm{m}} \sim 8-10 M_{\odot}\right)$.

If the core mass stays constant $\left(\sim 1.3 M_{\odot}\right)$, the degenerate core will simply cool like a white dwarf. In the core of a red-giant, the core mass grows because hydrogen and helium shell burning processes the material of the hydrogen-rich envelope into carbon-oxygen. (The helium layer has been dredged up by the penetrating surface convection zone when the layer expands by absorbing heat from the core. See Figure 20). In this sense, the core is essentially the same as accreting white divarfo.

\subsection{Collapse of $\mathrm{O}+\mathrm{Ne}+\mathrm{Mg}$ Core induced by Electron Captures}

When the core mass grows to $1.38 M_{0}$, the central density zeaches $4 \times 10^{\circ} \mathrm{g} \mathrm{cm}^{-3}$. Degenerate electrons provide another effect on stellar evolution, i.e., the electron Fermi energy exceeds the threshold for electron captures ${ }^{24} \mathrm{Mg}\left(\mathrm{e}^{-}, \nu\right)^{24} \mathrm{Na}\left(\mathrm{e}^{-}, \nu\right)^{24} \mathrm{Ne}$ and ${ }^{20} \mathrm{Ne}\left(\mathrm{e}^{-}, \nu\right)^{20} \mathrm{~F}\left(\mathrm{e}^{-}, \nu\right)^{20} \mathrm{O}$. The sesultant decrease in

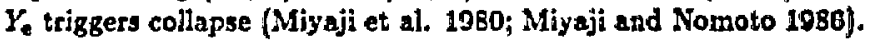

The hyojrodynamical behavior of collapse is somewhat diffetent from the iron core collapse of more massive stars (see, e.g., Hillebrandt et al. 1984; Burrows and Irattimer 1985; Hillebrandt 1986; Baron et al, 1986). The $\mathrm{O}+\mathrm{Ne}+\mathrm{Mg}$ core contains nuclear fuel which ignites during infall. Electron captures oceur only in the NSE layer behind the burning front and, therefore, the region of small $\boldsymbol{Y}_{e}$ is confined to a central Iegion which grows gradually. The collapse is slower than the collapse of the iron core of a massive star unitl the burning front has propagated to soughly $\sim 0.8 M_{\odot}$ (Hillebrandt et al. 1984). Afterwards the collapse accerelates quickly. $Y_{e}$ in the NSE region of the $O+N e+M g$ core is smaller than $Y_{e}$ in iron cores, because the entropy at the burning front is higher and thus the proton fraction is larger. These two effects result in a homologous core whose mass is smaller and an outer infalling layer which is less dense than is the case in iron core collpase. Such a structure has two effects on the bounce shock. First, the binding energy of the rebounding core is smaller and, hence, the shock wave in initially weaker (Brown et al. 1982; Lattimer et al. 1985). Secondly, the low density in the outer layers makes the shock propagation easier (Utillebrandt et al. 1984). Which effect dominates depends on the details of collapse hydrodyramics. 


\section{CONCLUDING REMARKS}

We have shown that the evolution and the final fate of stars is determined by their gravothermodynamical nature that depends on the equation of state and thus on the stellar moss. For messive sters, entropy in the interior is high and electrons are non-degenerate. Then the gravothurmalspecific heat is always nrgative and the star evolves toward higher density and temperature as the entropy in the conter decreases. Eventually nuclear statistical enuilibrium is realized through nuclear burning and the iron core formas. On the contrary, smaller mass stars have lower entropy so that electrons become degenerate at a certain stage of evolnaton. Then $c_{8}$ turns positive and the interior temperature starts to decrease as the star loser ertiong. These two types of evolution lead to two different type of collapse, i.e., the degererate core costains ruclear fuel while iron core does not. Between these two types, there is a mass range for which the star forms a sertidegenerate core. The evolution is complicated due to off-center aeon fiashes and thus careful calletation is required to know in which direction (degencrate or non-degenerate) the star evolves.

We note that the entropy structure at oxygen depletion of our $B M_{\odot}$ helium star model, which is equivalent to a $25 M_{\odot}$ star, is very similar to the old $25 M_{\odot}$ model by Weaer, Woosley, and Fuller (1085, WWF) who used the old ${ }^{12} \mathrm{C}(\alpha, \gamma){ }^{16} \mathrm{O}$ rate. Since the WWF $25 M_{\odot}$ star model forms a $1.35 M_{\odot}$ iron core, our model is expected to form a simjlar snall jron core despite our use of faster ${ }^{12} \mathrm{C}(\alpha, \gamma){ }^{18} \mathrm{O}$ reaction. This is because our carbon abundance after helium burnisg is about twice that of the latest $25 M_{\odot}$ model by Woosley and Weaver (1986a). Such a difference of carbon abundance may be due to differences in the treatment of convection.

We have emphasized the iruportance of the use of datailed ruclear reaction natwork before the oxygen exhaustion. If $Y_{0}$ in the silicon - sulfur layer is significantly small, it affects not only the wize of the jron core, but also ${ }^{50} \mathrm{Na}$ production during supernova explosion. Since the decays of ${ }^{50} \mathrm{Ni}$ and ${ }^{30} \mathrm{Co}$ into ${ }^{50} \mathrm{Fe}$ provide energy for the light curve, the ${ }^{56} \mathrm{Ni}$ mass is the crucial quantity to model the ligtht curve tail of Type II supernovae. It is particularly important for the Wolf-Rayet models of Type It supernovae, In the previous models, ${ }^{36} \mathrm{Ni}$ is synthesized when the sthock waye passes through silicon-rich layer (Weaver and Woosley 1080; Johnston and Yahil 1084). The density in the oxygen layer is too low to synthesize much ${ }^{56} \mathrm{Ni}$. If $Y_{0}<0.49$, which would be the case for smaller mass stars, the armount of 56 Ni would be small as expected from the NSE abundarce given as a function of $Y_{e}$ (Figure 21). Since $Y_{0}$ is smaller for smaller mass stars (Thielemann and Arnett 1985), we can obtain the lower mass limit of exploding stars that produce sufficiently large amount of ${ }^{56} \mathrm{Ni}$ for their light curves.

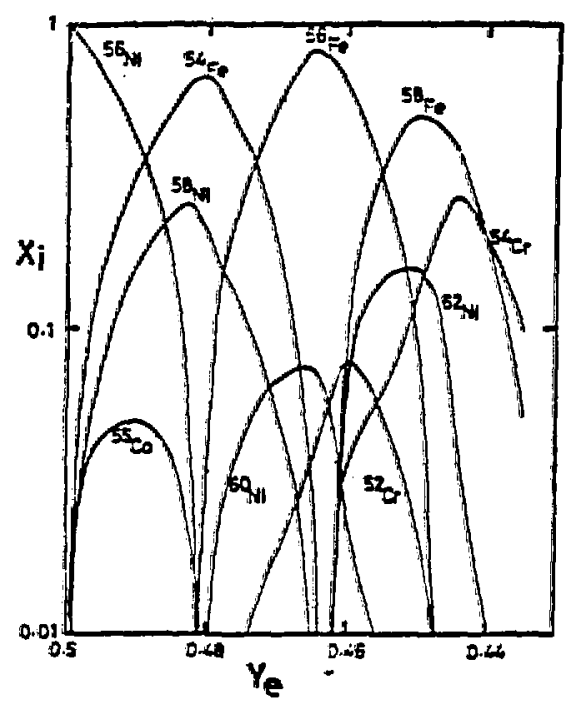

Figure 21: Nuclear statistical equilibrium aburdarce as a function of electron mole number, $Y_{\text {ee }}$, for $T=3 \times 10^{\circ} \mathrm{K}$ and $\rho=$ $1 \times 10^{7} \mathrm{~g} \mathrm{~cm}^{-3} . X_{i}$ denotes the mass fraction. 


\section{ACKNOWLEDGEMENT}

We would like to thark Dr. F.K. Thielemann for sending us his latest compilation of the nuclear reaction rates and Dr. E. Baron for the reading of the manuscript. It is a pleasure to thank Drs. S.F. Kahana, G.E. Brown, A. Yahil, A. Burrows, and J. Cooperstein for stimulating discussion and hospitality furing our stay in Brookhaven and Stony Brook. This work has been supported in part by the U. S. Department of Energy under Contract No. DE-AC02-76CH00010 and by the Japanese Ministry of Edueation, Science, and Culture through research grant nos. 59380001 and 60540152.

\section{REFERENCES}

Antonov, V.A. 1962, Vest. Leningr. Gos. Univ., 7, 135.

Ainett, W.D. 1972, Ap. J., 170, 681.

1978, in Physics and Astrophysics of Netstion Stars and Black Holes, ed. R. Giaconni and

R. Ruffini (Bologna: Soc. Italiana di Fisica), p.356.

Arnett, W.D., and Thielemann, F.-K. 1985, Ap. J., $295,589$.

Barkat, Z., Reiss, Y., and Rakavy, G. 1974, Ap. J. (Letters), 193, L21.

Baron, E., Cooperstein, J., and Kahama, S.H. 1986, private communication.

Brown, G.E., Bethe, H.A., and Baym, G. 1982, Nucl. Phys., A375, 481.

Burrows, A., and Lattimer, J.M. 1985, Ap. J. (Letters), 299, L19.

Caughlan, G.R., Fowler, W.A., Harris, M.J., and Zimmerman, B.A. 1985, Atomic Data and Nuclear Data Tables, 32, 197.

Doggett, L.B., and Branch, D. 1985, A. J., 00, 2303.

Fowler, W.A. 1984, Rev. Mod. Phys., 56, 149.

Fowler, W.A., Caughlan, G.R., and Zimmerman, B.A. 1975, Ann. Rev. Astr. Ap., 13, CD.

Fuller, G.M.; Fowler, W.A. and Newman, M. 1980, Ap. J. Suppl., 42, 447.

- 1982, Ap. J. Suppl., 48, 279.

Habets, G.M.F.J. 1985, Ph.D. Thesis, University of Amsterdarn.

- 1986, Astr. Ap., submitted.

Harris, M., Fowler, W.A., Caughlan, G.R., and Zimmerman, B.A. 1983, Ann. Rev, Astr. Ap., 21, 165.

Hashimoto, M., Hanawa, T., and Sugimoto, D. 1983, Pub. Astr. Soc. Japan, 35, 1.

Hashimoto, M., and Nomoto, K. 1986, in pzeparation.

Hayeshi, C., Hoshi, R., and Sugimoto, D. 1972, Prog. Theoret. Phys. Suppl., 22, 1.

Hillebrandt, W. 1986, this volume.

Hillebrandt, W., Nomoro, K., and Wolf, R.G. 1984, Astr. Ap., 133, 175.

Iben, I. Jr. 1986, this volume.

Ikeuchi, S., Nakazawa, Kr., Murai, T., Hoshi, R., and Hayashi, C. 1972, Prog. Theoret. Phys., 48. 1890.

Johnston, M.D., and Yahil, A. 1984, Ap. J., 285, 587.

Kahana, S.H. 1986, this volume.

Langer, N. 1986, Astr. Ap., submitted.

Lattimer, J.M., Burrows, A., and Yahil, A 1985, Ap. J., 288, 644.

Lynden-Bell, D., and Wood, R. 1968, M.N.R.A.S., 138, 495.

Miyaji, S., Nomoto, K., Yokoi, K., and Sugimoto, D. 1980, Pub. Astr. Soc. Japan, 32, 303

Milyaji, S., and Nomoto, K. 1986, Ap. J., submitted.

Nomoto, K. 1981, ia LA Symposium 93, Fundamental Problems in the Theory of Stellar Evolution, ed. D. Sugimoto, D.Q. Lamb, and D.N. Schramm (Dordrecht: Reidel), p.295.

—_. 1984a, Ap. J., 277, 791.

- 1984b, in Stellar Nucleosynthesis, ed. C. Chiosi and A. Renzini (Dordrecht: Reidel), p. 238.

Nomoto, K. 1986, Ann. N Y Acid. Sci., 470, 294.

Nomoto, K., Thielemann, F.K., and Yokoi, K. 1984, Ap. J., 286, 644.

Saio, H., and Nomoto, K. 1985, Astr. Ap., 150, L2L.

Schramm, D.X. 1986, this volume.

Sugimoto, D., Erjguchi, Y., and Hachisu, I. 1981, Ptog. Theoreu, Plys, Suppl, 70, 154. 
Sugimotn, D., and Nomoto, K. 1980, Sypace Sci. Rev, 25, 155.

Thielemann, F.-K. 1986, private communication.

Thielemann, F.-K., and Arnett, W.D. 1085, Ap. J., 2135,604.

Weaver, T.A., and Woosley, S.D. 108D, Ann. N Y Arad. Sei., 336, 335.

Weaver, T A., Wousley, S.E., and Tuller, G.M. 1985, in Nunerjeal Astroplugsics, ent. J. Centrella, J. LeBlane, and R. Buws (Porola, CA: Science 9 ook International), 13.374.

Weaver, T.A., Zinmermati, G,13., and Woosley, S.E. 1078, Ap. J., 22:, 1021.

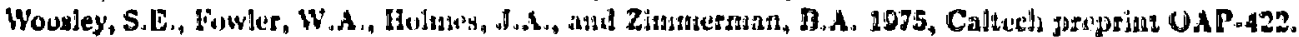

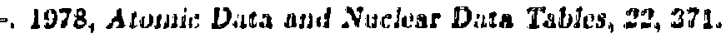

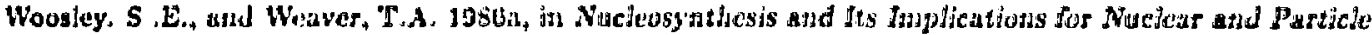
Physirs, ed. J. Awdouze and T. van Thuar (Dordrecht: Reidel).

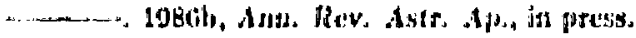

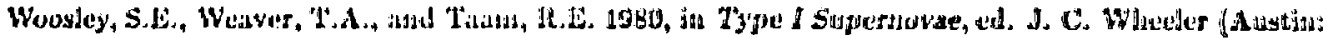
University of Texis), d). Dto. 